\title{
Simulation of neutron transmission performance of metallic spherical shell under temperature dependent neutron cross section
}

\author{
Yinghong Zuo ${ }^{1, *}$, Jinhui Zhu ${ }^{1, * *}$, and Honggang $\mathrm{Xie}^{1, * * *}$ \\ ${ }^{1}$ Northwest Institute of Nuclear Technology, 710024, Xi'an, China
}

\begin{abstract}
To study the effect of temperature dependent neutron cross section on the neutron transmission performance of typical metallic spherical shells, this paper generated the temperature dependent neutron cross section data by using NJOY program, and established the computational model of interaction of neutron with iron and aluminium spherical shell. The temperature evolution of energy spectrum of transmitted neutron and secondary gamma ray, and the number ratio of transmitted neutrons to secondary gamma rays has been studied at various neutron energies. The results show that only when the energies of the incident neutron are in a certain range, the temperature dependent cross sections can have a significant effect on the energy spectrum of the rays penetrating the spherical shell. For different metal materials, the transmitted neutron spectrum and the secondary gamma ray spectrum will be significantly affected when the temperature increases to different high value. Generally, the transmitted neutron number decreases with the increase of temperature, and the number ratio of transmitted neutron to secondary gamma ray also decreases with the increase of temperature, while the changing trend of number of secondary gamma ray with temperature is related to the type of shell material.
\end{abstract}

\section{Introduction}

The energy-related nuclear cross-section data is indispensable when the Monte Carlo program MCNP is used to do a simulation of particle transport [1]. However, for most of the nuclides in the MCNP program, only the thermal cross section data at room temperature $(300 \mathrm{~K})$ are available; for a few nuclides, the thermal cross section data at multiple temperatures are available [2]. In temperaturedependent particle transport simulation, for most nuclides without thermal cross section, MCNP program only modifies the elastic scattering cross section of the thermal part by Maxwell temperature [3-4]. To obtain more accurate simulation results of temperature-dependent particle transport using MCNP program, it is necessary to develop a temperature-dependent neutron cross section database.

NJOY [5], which is widely used in the world, can process the original ENDF [6-7] format evaluation database into nuclear cross section data for different simulation programs such as MCNP, ANISN and WIMS. For MCNP program, the neutron cross section data in A Compact ENDF format can be produced by using NJOY program. Most of the previous literature [8-9] focused on the nuclear reactor problem, so the temperature range of the temperature-dependent section library is narrow, only between $300 \mathrm{~K}$ and $2000 \mathrm{~K}$. In order to study the rules of interaction between radiation and matters at higher temperatures, i.e. when nuclear materials are in plasma state, the temperature-dependent neutron cross section data is

\footnotetext{
*e-mail: zuoyinghong@nint.at.cn

**e-mail: zhujinhui@nint.ac.cn

***e-mail: xiehonggang@nint.ac.cn
}

developed based on the evaluation database ENDF/B-VII [6] and CENDL-3.1 by using NJOY program. Taking the spherical shells made of $\mathrm{Fe}$ and aluminum as examples, the distribution of neutron energy spectra and secondary gamma energy spectra through the shells under temperature-dependent neutron cross sections is studied

\section{Physical model and method}

The simplified physical model is established as shown in figure 1. The neutron source is located at the spherical center of the vacuum sphere with a radius of $10 \mathrm{~cm}$. The vacuum sphere is surrounded by a metallic spherical shell. The shell material is iron or aluminum, which are commonly used in nuclear radiation physics. Neutron source located at the center of the sphere emits neutrons isotropically, that thereafter transporting through the spherical shell materials. When neutrons interact with atoms in a spherical shell, they may be absorbed or penetrated.

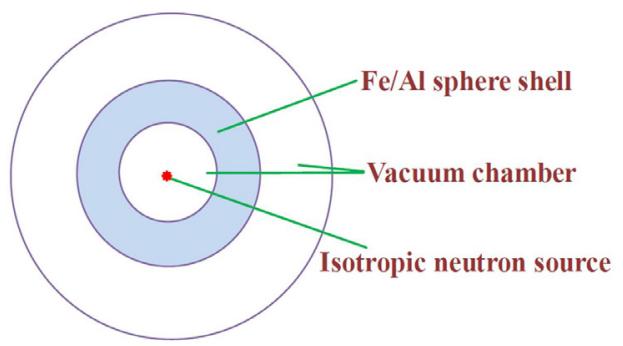

Figure 1. Physical model of spherical shell 
The temperature of the spherical shell varies from 100 $\mathrm{K}$ to $10^{8} \mathrm{~K}$. When the temperature far exceeds the melting point of the material, the material will become plasma state. The temperature dependent cross section data are processed by using NJOY program and the cross section data at $300 \mathrm{~K}$ is directly derived from the cross section database of MCNP program.

For the spherical shell model shown in figure 1, the MCNP4C program and the F1 tally card are used to calculate the spectrum of the secondary gamma-ray and the number ratio of the transmitted neutron to the secondary gamma ray of the isotropic neutron source interacting with iron or aluminum spherical shells with different thicknesses. The weights of neutron source are 1, indicating unit source strength. The simulated number of particles is $1 \times 10^{8}$.

\section{Simulation results and discussions}

\subsection{Interaction between monoenergy neutron and Fe spherical shell}

Figure 2 shows the total cross section and secondary gamma ray production cross section of neutrons interacting with $\mathrm{Fe}$ at different temperatures. As shown in figure 2 , when the incident neutron energy exceeds $1 \mathrm{MeV}$, the temperature has little effect on the total cross section and secondary gamma ray production cross section. The influence of temperature on the cross section data is different with different incident neutron energy, and the influence of temperature on the total cross section and the secondary gamma ray production cross section is also different.
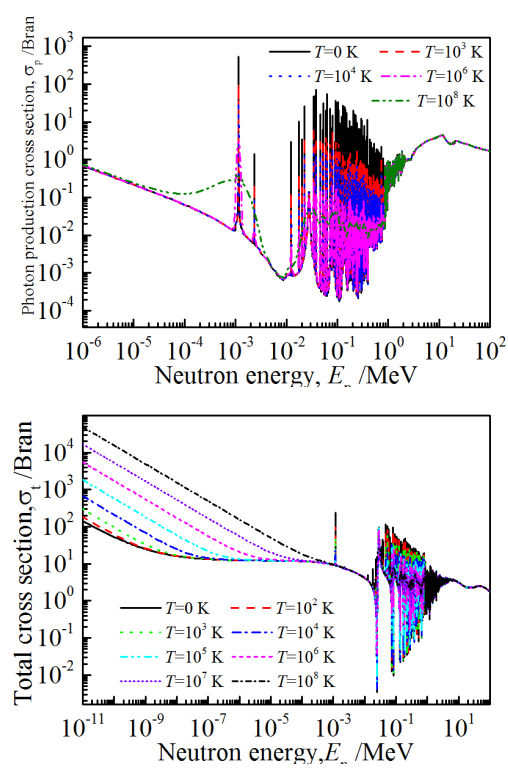

Figure 2. Total cross section and photon generation cross section of neutron-iron interaction at different temperatures

According to the equivalent relationship between energy and temperature, $1 \mathrm{eV}$ corresponds to $11600 \mathrm{~K}$, and $0.1 \mathrm{MeV}$ corresponds to $10^{9} \mathrm{~K}$. When the energy of source neutron are $1 \mathrm{MeV}$ or $14 \mathrm{MeV}$, and the temperature of the iron spherical shell varies from $0 \mathrm{~K}$ to $10^{6} \mathrm{~K}$, the change of temperature has little effect on the energy spectrum of the transmitted neutron and the energy spectrum of the secondary gamma ray. The thickness of iron spherical shell is $2 \mathrm{~cm}$ in the simulation model. Figure 3 is the simulated energy spectrum distribution of transmission neutron and secondary gamma ray at different temperatures when the energy of incident neutron is $0.1 \mathrm{MeV}$.
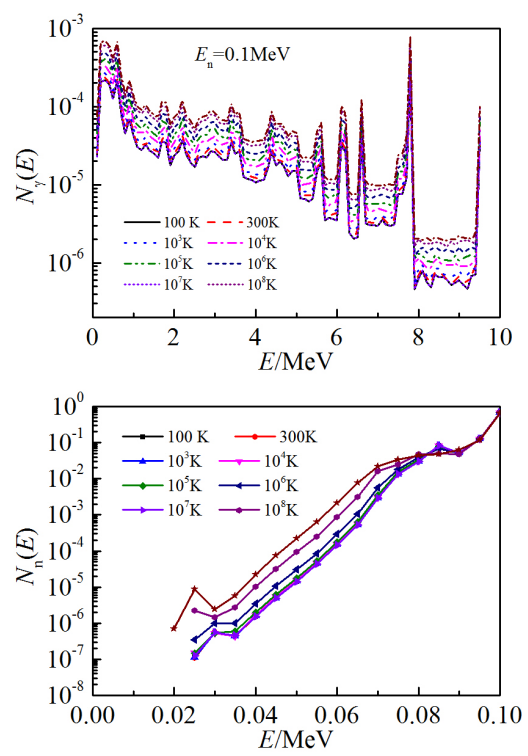

Figure 3. The energy spectrum distribution of secondary gamma ray and transmitted neutron at the case of iron spherical shell and incident neutron energy of $0.1 \mathrm{MeV}$

As can be seen from figure 4, when the incident neutron energy is $0.1 \mathrm{MeV}$, the number of transmitted neutrons is slightly less than 1 , that is, the number of neutrons absorbed by iron spherical shell is very small, the number of transmitted neutrons decreases slightly with the increase of iron temperature, while the number of secondary gamma ray increases with the increase of iron temperature, so the value of $N_{n} / N_{\gamma}$ decreases with the increase of iron temperature. According to the above calculation without considering the influence of temperature on the cross section of neutron-iron interaction, when the incident neutron energy is less than $1 \mathrm{MeV}$, the number of secondary gamma rays generated is very small, resulting in the value of $N_{n} / N_{\gamma}$ more than 100 .

\subsection{Interaction between monoenergy neutron and aluminum spherical shell}

In order to compare with the calculated results of iron spherical shell, the incident neutron energy is $0.1 \mathrm{MeV}$ and the thickness of aluminum spherical shell is $2 \mathrm{~cm}$.

From figure 5, we can see that the temperature has a great influence on the energy spectrum of transmitted neutrons and secondary gamma rays. For the neutron spectrum, when the temperature is less than $10^{4} \mathrm{~K}$, the transmission neutron spectrum curve coincides, which shows that the energy spectrum distribution is very little affected 

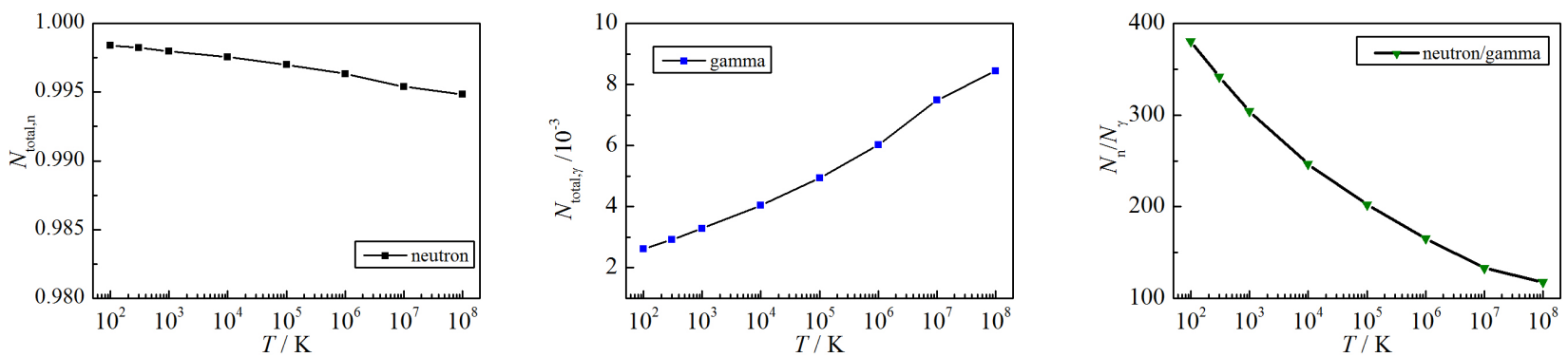

Figure 4. The number of transmission neutron, secondary gamma ray and their ratios versus temperature when the incident neutron energy is $0.1 \mathrm{MeV}$ and spherical shell material is iron
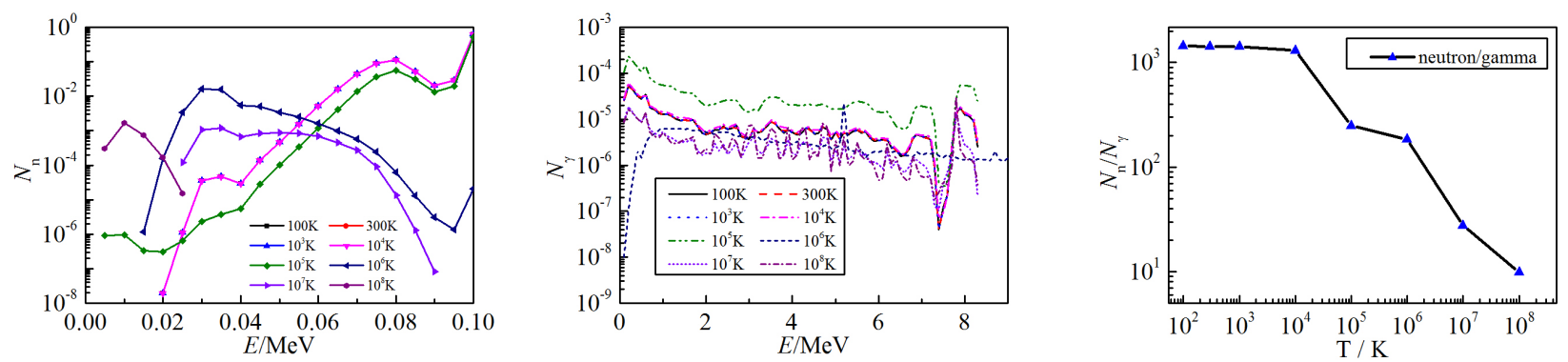

Figure 5. The left two is energy spectrum of transmitted neutron and secondary gamma ray of aluminum spherical shell at incident neutron energy of $0.1 \mathrm{MeV}$; the third is the ratio $N_{n} / N_{\gamma}$ versus $\mathrm{T}$ when $E_{n}=0.1 \mathrm{MeV}$ for aluminum shell

by temperature, but when the temperature of aluminum spherical shell exceeds $10^{4} \mathrm{~K}$, the transmission neutron spectrum varies greatly.Figure 5 also shows that the number ratio of transmitted neutrons to secondary gamma rays decreases with the increase of temperature when the incident neutron energy is $0.1 \mathrm{MeV}$.

\subsection{Interaction between fission neutron source and metallic spherical shell}

When the fission neutron is incident into an iron spherical shell with thickness of $2 \mathrm{~cm}$, as shown in figure 6 , the number of transmitted neutrons decreases slightly with the increase of temperature, while the number of secondary gamma rays increases with the increase of temperature. As the number of neutrons decreases and the number of secondary gamma rays increases, the ratio of transmission neutrons to secondary gamma rays decreases with the increase of temperature, but the range of variation is very small. The value of ratio decreases from 37.8 to 33.1 when the temperature increases from $100 \mathrm{~K}$ to $10^{8} \mathrm{~K}$.

Figure 7 is the spectra of transmission neutron and secondary gamma-ray when the fission neutron spectrum is incident into aluminum spherical shell with thickness of $2 \mathrm{~cm}$. From figure 7, it can be seen that when the temperature changes from $100 \mathrm{~K}$ to $10^{4} \mathrm{~K}$, the spectra of the transmission neutrons at different temperature coincide basically. When the temperature of aluminum spherical shell exceeds $10^{4} \mathrm{~K}$, the spectra of transmission neutron change greatly with the increase of temperature. When the temperature is below $10^{4} \mathrm{~K}$, the temperature has little effect on the spectrum of secondary gamma ray. When the temperature exceeds $10^{4} \mathrm{~K}$, the distribution of the secondary gamma ray spectrum is greatly affected by temperature, but the energy corresponding to the peaks below $10 \mathrm{MeV}$ does not change. By comparing the spectra of transmission neutron and secondary gamma ray of $\mathrm{Fe}$ and aluminum spherical shells, the results show that for $\mathrm{Fe}$ and $\mathrm{Al}$, the temperature needs to rise to different thresholds to have a significant effect on the transmission spectrum.

Figure 8 shows the temperature dependence of number of transmitted neutrons, secondary gamma rays and their ratios when the fission neutron spectrum is incident to an aluminum spherical shell with thickness of $2 \mathrm{~cm}$. The number of transmitted neutrons, and the number of secondary gamma rays both decreases with the increase of temperature. When the temperature is less than $10^{4}$ $\mathrm{K}$, the number of transmitted neutrons and the number of secondary gamma rays are little affected by temperature; when the temperature is higher than $10^{4} \mathrm{~K}$, both the number of transmitted neutrons and the number of secondary gamma rays decrease rapidly with the increase of temperature, and the number ratio of neutrons to secondary gamma rays decreases with the increase of temperature.

\section{Conclusions}

Based on MCNP and NJOY program, the neutron transmission properties of metallic spherical shells under temperature-dependent neutron cross section are studied. The following conclusions are drawn: (1) The influence of temperature on neutron cross section data is different 

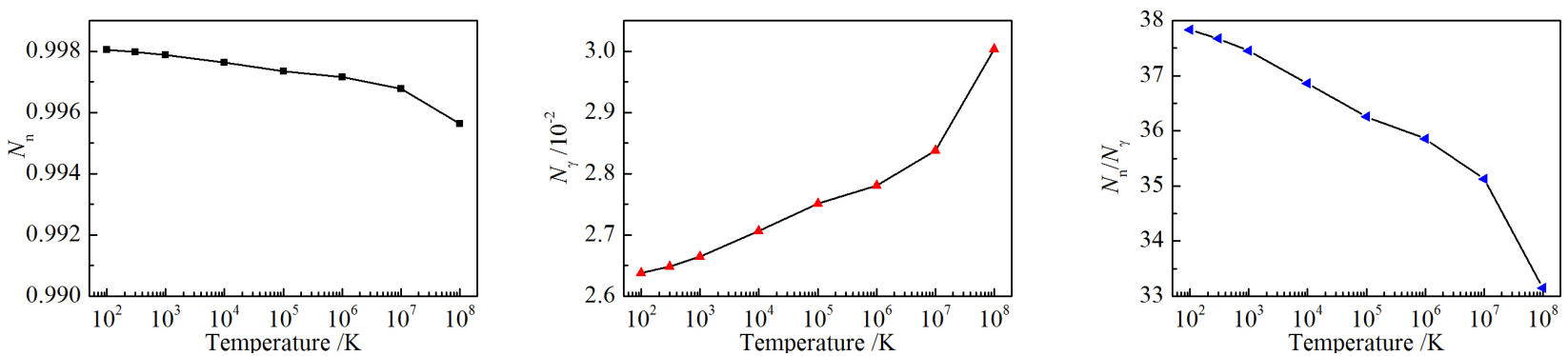

Figure 6. The number of transmission neutron, secondary gamma ray and their ratios versus temperature for iron spherical shell induced by fission neutron source
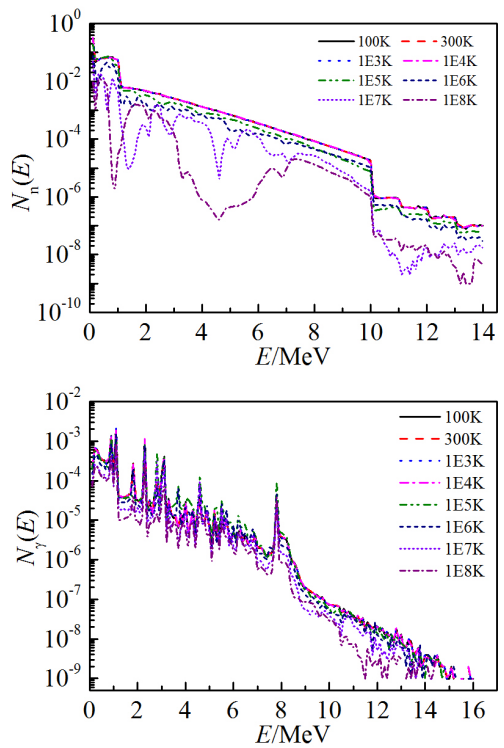

Figure 7. Spectra of transmission neutron and secondary gamma rays of $\mathrm{Al}$ spherical shell induced by fission neutron source

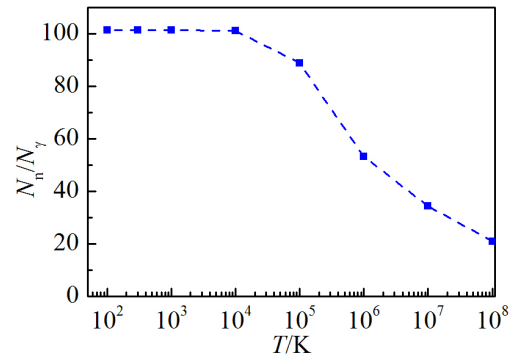

Figure 8. The ratio $N_{n} / N_{\gamma}$ versus $T$ for aluminum spherical shell induced by fission neutron source

in different neutron energy intervals. For example, when the neutron energy is between $0.01 \mathrm{MeV}$ and $1 \mathrm{MeV}$, the change of temperature has a great effect on the total cross section and gamma ray production cross section of the in- teraction between neutrons and iron atoms, while it will has little effect on the cross section when the neutron energy exceeds $1 \mathrm{MeV}$. (2) For the spherical shell of iron or aluminium with thickness of $2 \mathrm{~cm}$, the number of transmitted neutrons decreases slightly with the increase of temperature, and the number ratio of transmitted neutrons to secondary gamma rays decreases with the increase of temperature when the incident neutron energy is $0.1 \mathrm{MeV}$. (3) When neutron source is the fission spectrum with an average energy of about $2.1 \mathrm{MeV}$, the transmitted neutron number, and the number ratio of transmitted neutron to secondary gamma ray both decrease with the increase of temperature for iron and aluminium spherical shell. The variation trend of the number of secondary gamma ray with temperature is related to the type of spherical shell material. For iron, it increases with the increase of temperature, and for Al, it decreases with the increase of temperature.

\section{References}

[1] Briesmeister J F. (Los Alamos Scientific Laboratory, New Mexico, 1997) LA-12625-M.

[2] Chai Xiaoming, Wang Kan, Yu Ganglin. Nuclear Physics Review, 23( 2), 111-114 (2006)

[3] Xie Zhongsheng, Deng Li. Neutron Transport Theory and Computational Methods. (Northwestern Polytechnical University Press,Xi'an, 2015)

[4] Zhu Jinhui, Zhuo Jun, Tao Yinglong, et al. High Power Laser and Particle Beams, 24(8), 1985-1989 (2012)

[5] MacFarlane R E, Muir D W. Los Alamos Laboratory Report LA-12740-M. 1994

[6] Chadwick M B, Oblozinsky P, Ermanm H, et al. Nuclear Data Sheets, 107, 2931-3060 (2006)

[7] Macfarlane R E,Kahler A C. Nuclear Data Sheets, 111(12), 2739-2890 (2010)

[8] Zou Yang. Nuclear Power Engineering, 33(3), 12-16 (2012)

[9] Li Songyang, Wang Kan, Yu Ganglin. Atomic Energy Science and Technology, 43(5), 385-388 (2009) 